\title{
Spatial distribution of the gamma-ray bursts at very high redshift
}

\author{
Attila Mészáros \\ Charles University, Faculty of Mathematics and Physics, Astronomical Institute, \\ V Holešovičkách 2, Prague 8, Czech Republic, \\ email:meszaros@cesnet.cz
}

\begin{abstract}
The author - with his collaborators - already in years 1995-96 have shown - purely from the analyses of the observations - that the gamma-ray bursts (GRBs) can be till redshift 20. Since that time several other statistical studies of the spatial distribution of GRBs were provided. Remarkable conclusions concerning the star-formation rate and the validity of the cosmological principle were obtained about the regions of the cosmic dawn. In this contribution these efforts are surveyed.
\end{abstract}

Keywords. Cosmology: large-scale structure of the Universe, observations, miscellaneous; Stars: gamma-ray bursts

\section{Introduction}

It is a standard cosmology that the observable part of the Universe is finite and has the size of $\sim(10-20) \mathrm{Gpc}$, if one uses the so called "proper-motion distance". The redshifts of the observed objects can be - in principle - arbitrarily large, but for $z \rightarrow \infty$ the limiting proper-motion distance remains finite, and its value depends on the cosmological omega parameters and on the Hubble constant. The relevant exact formulas can be found, e.g., in Weinberg (1972) and Carroll et al. (1992).

The Cosmological Principle requires that the Universe be spatially homogeneous and isotropic on scales larger than the size of any structure (Peebles (1993)). Exactly, it is said on the page 15 of this book that "...in the large scale average the visible parts of our universe are isotropic and homogeneous". But - trivially - the averaging should happen far below the $\sim(10-20)$ Gpc scales. In other words, there should exist a transition scale not larger than, say, $\sim 1 \mathrm{Gpc}$, and above this one no structures should exist.

Yadav et al. (2010) means that this transition scale is $\simeq 260 h^{-1} \mathrm{Mpc}$, where $h$ is the Hubble-constant in unit $100 \mathrm{~km} /(\mathrm{sMpc})$, and the Cosmological Principle holds. But, oppositely, there are publications claiming supports for the structures with $\sim$ Gpc sizes. For example, Collins \& Hawking (1973) and Birch (1982) speak about a possible global rotation in the observable part of the Universe. Other observations (cf. Rudnick et al. (2007)) claim the existence of structure with size $\sim 140 \mathrm{Mpc}$, but at redshift around 1 . A recent publication about the spatial distribution of quasars (Clowes et al. (2013)) claims the existence of a structure with a scale $>1 \mathrm{Gpc}$.

Generaly speaking, any observational result from the high redshifts regions of the Universe is highly useful both from the astrophysical and cosmological point of views. Astrophysically, one can obtain some observational reflections, e.g., about the reionization era; cosmologically, one an verify by observations the fulfilment of the Cosmological Principle. In this contribution the statistical studies of the spatial distribution of gamma-ray bursts (GRBs) - done mainly by the author and his coauthors - are briefly summarized. 


\section{GRBs: Discovery, brief history and the diversity}

The first GRB was detected in year 1967; the first artice about the discovery of 16 GRBs was published in 1973 (Klebesadel et al. (1973)). In period 1973-1990 $2(10-20)$ GRBs were detected annually. Already in 1981 it was found that there are two different types of GRBs separable by the observed duration. There are short and long GRBs. The rough separating limit is at duration $\simeq 2 \mathrm{sec}$ (Mazets et al. (1981)). In years 19902000 the BATSE instrument on the Compton Gamma-Ray Observatory increased the number of detected GRBs and confirmed the short-long separation (see, e.g., Meegan et al. (1992), Goldstein et al. (2013) and the references therein).

At year 1997 a long GRB was detected also at other photon energy bands, because the so-called "afterglow" was followed after the discovery of the BeppoSAX satellite (Costa et al. (1997)). After that, at the coming years, it was observationally confirmed that the long GRBs are connected to supernovae (for details see, e.g., Woosley \& Bloom (2006)). For the short ones only in 2013 came the observational support that they are given by the merging two neutron stars (black holes) forming macronovae (Tanvir et al. (2013)). The simultaneous detection of the gravitational wave and macronova from 17 August 2017 confirmed that the observed gravitational waves and the short GRBs should have common origin (Abbott et al. (2017)).

It has to be noted that there are several statistical studies claiming that - beyond the short and long ones - also other subgroups exists for GRBs (for details and other issues see, e.g., Levan et al. (2014), Řípa \& Mészáros (2016) and the references therein).

\section{GRBs: Redshifts}

Probably the first article about the redshifts of GRBs was presented by Usov \& Chibisov (1975). Paczyński (1986) has shown that GRBs should be at $z \simeq(1-2)$. In years 1995-97 the author and his coauthors confirmed the Paczyński's conclusion and have shown that GRB can be till $z \simeq 20$ (Mészáros \& Mészáros (1995), Mészáros \& Mészáros (1996), Horváth et al. (1996), Reichart \& Mészáros (1997)). It was also shown that mainly the long GRBs should follow the star-formation-rate (Mészáros et al. (2006)).

Add here that only a small fraction of GRBs has directly measured redshifts from the afterglows (for a detailed list about the redshifts see, e.g., Perley (2017)).

\section{GRBs: Anisotropies in the sky distribution}

The first clear indirect observational proof for the cosmological origin of GRBs was given by Meegan et al. (1992). There was no concentration on the sky positions of the observed GRBs toward the Galactical plane. This indirect support of the cosmological origin was then strongly formulated by Tegmark et al. (1996). This study did not find any concentration toward the Galactical plane and did not find deviation from the isotropic celestial distribution.

Balázs et al. (1998) accepted immediately the cosmological origin of GRBs, and hence did not search for any concentration toward the Galactical plane. It studied generally by statistical tests the isotropy of the sky distribution. In essence, it tested the fulfilement of The Cosmological Principle, because - if fulfilled - the distribution must remain isotropic. Balázs et al. (1998) clearly claimed first that the sky distribution of short BATSE's GRBs was not isotropic. This proclaim was then confirmed by several other articles of the author and his collaborators (see Balázs et al. (1999), Mészáros et al. (2000a), Vavrek et al. (2008)). In addition, both the BATSE's intermediate and long subclasses were 


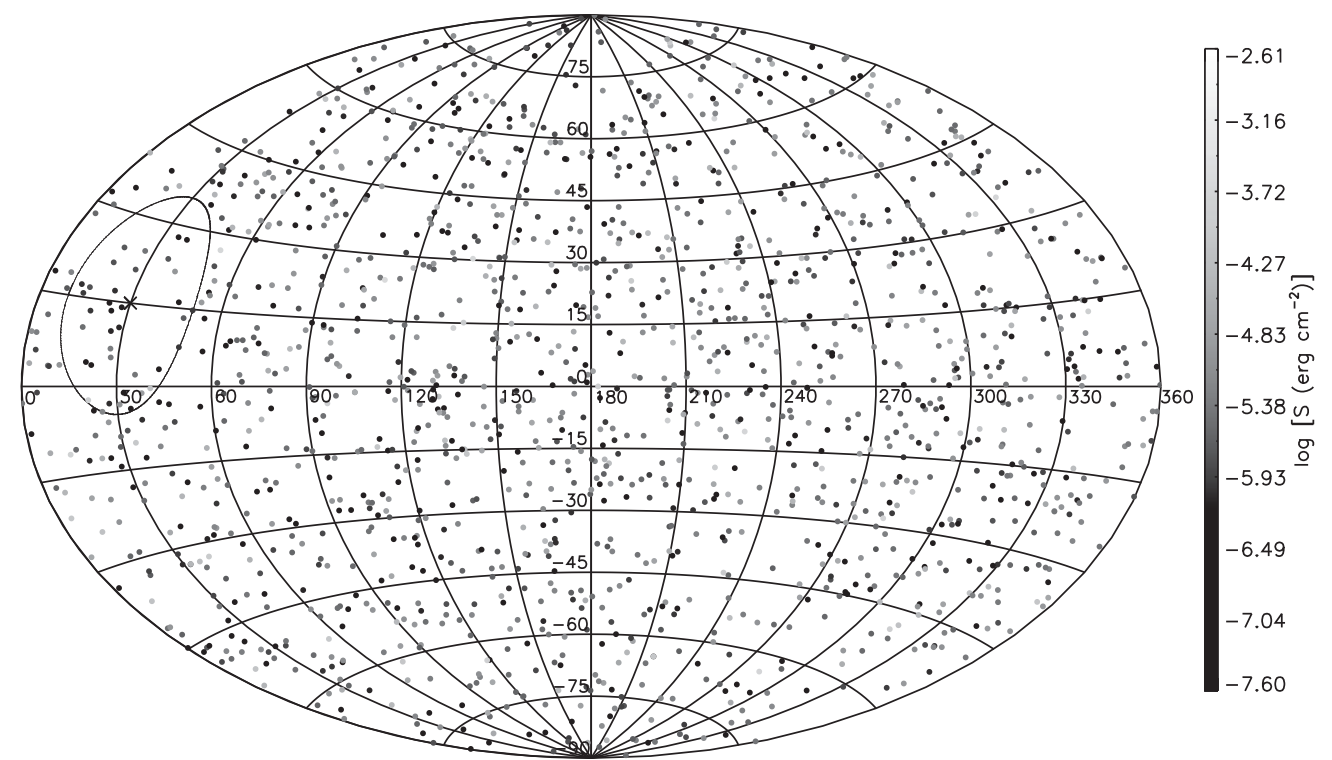

Figure 1. Sky distribution of Fermi 1591 GRBs in Galactical coordinates. The area around $l=30^{\circ}, b=15^{\circ}$ with a radius $20^{\circ}$ shows some deviaton from the isotropy in the value of the fluence (time integrated flux) denoted as $S$ - for more details see Ŕípa \& Shafieloo (2017). [Credit J.Řípa]

found to be distributed also anisotropically (see Mészáros et al. (2000b), Mészáros \& Štoček (2003)) and Vavrek et al. (2008) for more details). After Vavrek et al. (2008) the existence of the Gpc structures and thus the problems of the Cosmological Principle were declared by Mészáros et al. (2009a) and Mészáros et al. (2009b). All these 2D studies were based on the BATSE data, because in the BATSE dataset only few GRBs had measured redshifts (for details see Bagoly et al. (2003) and Mészáros et al. (2011)).

Recently two articles obtained similar results based on the dataset of the Fermi satellite. Rípa \& Shafieloo (2017) - using the the whole dataset - found a noticeable deviation from the randomness for a given part of sky (see Figure 1). At the same dataset Tarnopolski (2017) found an anisotropy separately for the short subclass; for the long subclass the assumption of isotropy was not rejected by his tests.

\section{GRBs: 3D statistical studies}

For the limited sample of GRBs, which have directly measured redshifts from the afterglows, the study of 3D structures became also possible, Only a small fraction of GRBs have directly measured redshifts (Perley (2017)), and hence selection effects can play an important role here. In addition, in the topic of GRBs it is not sure that the apparently fainter GRBs are at higher redshifts (Mészáros et al. (2011)). Under these conditions spatial structures on the Gpc scales were found (Horváth et al. 2014, Horváth et al. 2015, Balázs et al. 2015, Sokolov et al. 2015, Verkhodanov et al. 2015, Bagoly et al. 2016a, Bagoly et al. 2016b).

\section{Implications}

GRBs are partly at huge redshits. This means that they may well serve as natural springs of observations from the dawn of the Universe. 


\section{References}

Abbott, B. P. et al. 2017 ApJL, 848, L13

Bagoly, Z. et al. 2003, A\&A, 398, 919

Bagoly, Z. et al. 2016a, Galaxies at High Redshift and Their Evolution Over Cosmic Time, IAU Symp. 319, 2

Bagoly, Z. et al. 2016b, Galaxies at High Redshift and Their Evolution Over Cosmic Time, IAU Symp. 319, 3

Balázs, L. G., Mészáros, A., \& Horváth, I. 1998, A\&A, 339, 1

Balázs, L. G. et al. 1999, A\&SAS, 138, 417

Balázs, L. G. et al. 2015, MNRAS, 452, 2236

Birch, P. 1982, Nature, 298, 451

Carroll, S. M., Press, W. H., \& Turner, E. L. 1992, ARAA, 30, 499

Clowes, R. G. et al. 2013, MNRAS, 429, 2910

Collins, C. B. \& Hawking, S. W. 1973, MNRAS, 162, 307

Costa, E. et al. 1997, Nature, 387, 783

Goldstein, A. et al. 2013, ApJS, 208, 21

Horváth, I., Mészáros, P., \& Mészáros, A. 1996, ApJ, 470, 56

Horváth, I., Hakkila, J., \& Bagoly, Z. 2014, A\&A, 561, L12

Horváth, I. et al. 2015, A\& A, 584, A48

Klebesadel, R. W., Strong, I. B., \& Olson, R. A. 1973, ApJ, 182, L85

Levan, A. J. et al. 2014, ApJ, 781, 13

Mazets, E. P. et al. 1981, ApשSSS, 80, 3

Meegan, C. A. et al. 1992, Nature, 355, 143

Mészáros, A. \& Mészáros, P. 1996, ApJ, 466, 29

Mészáros, A., Bagoly, Z,, \& Vavrek, R. 2000a, A 6 A, 354, 1

Mészáros, A. et al. 2000b, ApJ, 539, 98

Mészáros, A. \& Štoček, J. 2003, A\& A, 403, 443

Mészáros, A. et al. 2006 A $\varangle A, 455,785$

Mészáros A. et al. 2009a, Baltic Astronomy, 18, 293

Mészáros, A. et al. 2009b, Sixth Huntsville GRB Symposium, AIP Conf. Proc., 1133, 483

Mészáros, A. et al. 2011, A\&SA, 529, A55

Mészáros, P. \& Mészáros, A. 1995, ApJ, 49, 9

Paczyński, B. 1986, ApJL, 308, L43

Perley, D. 2017, http://www.astro.caltech.edu/grbox/grbox.php

Peebles, P. J. E. 1993, Principles of Physical Cosmology (Princeton University Press)

Reichart, D. E. \& Mészáros, P. 1997, ApJ, 483, 597

Ř́pa, J. \& Mészáros, A. 2016, Ap\&SSS, 361, 370

Ř́pa, J. \& Shafieloo, A. 2017, ApJ, 851, 15

Rudnick, L., Brown, S., \& Williams, L. R. 2007, ApJ, 671, 40; Errata: ApJ, 678, 1531 (2008)

Sokolov, I. V. et al. 2015, Quark Phase Transition in Compact Objects, SAO, Russia, 111

Tanvir, N. R. et al. 2013, Nature, 500, 537

Tarnopolski, M. 2017, MNRAS, 472, 4819

Tegmark, M. et al. 1996, ApJ, 468, 214

Usov, N. V. \& Chibisov, G. V. 1975, Soviet Astronomy, 19, 115

Vavrek, R. et al. 2008, MNRAS, 391, 1741

Verkhodanov, O. V. et al. 2015, Quark Phase Transition in Compact Objects, SAO, Russia, 142

Weinberg, S. 1972, Gravitation and Cosmology (J.Wiley, New York - London - Sydney - Toronto)

Woosley, S. E. \& Bloom, J. S. 2006, ARAA, 44, 507

Yadav, J. K., Bagla, J. S., \& Khandai, N. 2010, MNRAS, 405, 2009 